\title{
8-2018
}

\section{Relation between Sexual and Gender Minority Status and Suicide Attempts Among Veterans Seeking Treatment for Military Sexual Trauma}

\author{
Minden B. Sexton \\ Margaret T. Davis \\ RaeAnn E. Anderson \\ University of North Dakota, raeann.anderson@UND.edu \\ Diana C. Bennett \\ Erin Sparapani
}

See next page for additional authors

\section{How does access to this work benefit you? Let us know!}

Follow this and additional works at: https://commons.und.edu/psych-fac

Part of the Psychology Commons

\section{Recommended Citation}

Minden B. Sexton, Margaret T. Davis, RaeAnn E. Anderson, et al.. "Relation between Sexual and Gender Minority Status and Suicide Attempts Among Veterans Seeking Treatment for Military Sexual Trauma" (2018). Psychology Faculty Publications. 25.

https://commons.und.edu/psych-fac/25

This Article is brought to you for free and open access by the Department of Psychology at UND Scholarly Commons. It has been accepted for inclusion in Psychology Faculty Publications by an authorized administrator of UND Scholarly Commons. For more information, please contact und.commons@library.und.edu. 
Authors

Minden B. Sexton, Margaret T. Davis, RaeAnn E. Anderson, Diana C. Bennett, Erin Sparapani, and Katherine E. Porter 


\section{SUICIDALITY: SEXUAL AND GENDER MINORITY CONSIDERATIONS}

Relation between Sexual and Gender Minority Status and Suicide Attempts Among Veterans Seeking Treatment for Military Sexual Trauma

Minden B. Sexton ${ }^{*}, 1,2$

Margaret T. Davis ${ }^{3}$

RaeAnn E. Anderson ${ }^{4}$

Diana C. Bennett ${ }^{1,2,5}$

Erin Sparapani ${ }^{1,2,6}$

Katherine E. Porter ${ }^{1,2}$

${ }^{1}$ Mental Health Service, Ann Arbor Veterans Healthcare System, Ann Arbor, Michigan

${ }^{2}$ Department of Psychiatry, University of Michigan Medical School, Ann Arbor, Michigan

${ }^{3}$ Yale University School of Medicine, Department of Radiology and Biomedical Imaging.

${ }^{4}$ Department of Psychological Sciences, Kent State University, Kent, Ohio

${ }^{5}$ Behavioral Health Care Line, New Mexico VA Healthcare System, Albuquerque, New

Mexico

${ }^{6}$ Cognitive Behavioral Consultants, White Plains, NY

RUNNING HEAD: SUICIDALITY: SEXUAL AND GENDER MINORITY CONSIDERATIONS 


\section{SUICIDALITY: SEXUAL AND GENDER MINORITY CONSIDERATIONS}

*Corresponding author: Minden B. Sexton, Ph.D. Ann Arbor Veterans Healthcare

System, Mental Health Service (116C), 2215 Fuller Rd., Ann Arbor, 48105.

Minden.Sexton@va.gov. 734-222-7462.

Role of Funding Source: This study was supported by the Ann Arbor Veterans

Healthcare System Mental Health Service and the University of Michigan Department of

Psychiatry. The contents do not necessarily represent the views of the U.S. Department of Veterans Affairs or the United States Government. 


\title{
SUICIDALITY: SEXUAL AND GENDER MINORITY CONSIDERATIONS
}

\begin{abstract}
There is limited study of suicidal behaviors among Veterans identifying as sexual and gender minorities (SGM) despite previous research indicating rates of suicide attempts are high within civilian SGM populations. Further, some research incorporating military service members suggests those identifying as SGM are disproportionately exposed to military sexual trauma (MST), an additional risk factor for negative psychiatric sequelae. To address healthcare research disparities among minority Veterans (i.e., women, those endorsing MST, SGM), we examined presentations of Veterans $(N=277)$ who attended initial consultation appointments for MST-related treatment and completed a semistructured clinical interview including demographic characteristics, history of suicide attempts (HSA), and a diagnostic evaluation. Twenty-eight (10.1\%) Veterans identified as SGM. SGM/non-SGM groups were contrasted on suicidal and psychiatric morbidity outcomes. Overall, endorsement of HSA was high (30.7\%). Despite similar clinical profiles, $53.6 \%$ of Veterans who identified as SGM endorsed HSA in contrast with $28.1 \%$ of peers identifying as heterosexual and non-transgender, a significant effect of small-tomoderate size. Findings suggest assessment and clinical management of suicidality is of critical importance for clinicians providing services to Veterans pursuing recovery from MST, generally, and may be especially so when delivering care to sexual and gender minorities. Further, results underscore the need for culturally competent delivery of trauma-focused interventions.
\end{abstract}

Keywords: suicide attempts; Veterans; military sexual trauma; sexual minorities; gender minorities 


\section{SUICIDALITY: SEXUAL AND GENDER MINORITY CONSIDERATIONS}

\section{Introduction}

Veteran suicide is a significant public health concern, with mounting evidence suggesting that Veterans are at higher risk for both attempt and death by suicide than civilian peers (Huguet, Kaplan \& Bossarte., 2014). Currently, suicide has risen to the second leading cause of death among United States Veterans of both genders (Weiner, Richmond, Conigliaro \& Wiebe., 2011). The continued rise in Veteran suicide rates despite extensive risk prevention efforts highlights the urgent need for research focused on how and why suicidal behavior develops in this population. This study examines the histories of suicide attempts and psychiatric correlates among sexual and gender minority (SGM) Veterans seeking military sexual trauma (MST)-related treatment in comparison to their non-SGM peers.

\section{PTSD and MST as Risk Factors for Suicidality}

Posttraumatic Stress Disorder (PTSD) has been linked to all forms of suicidal behavior (e.g. ideation, attempts, death by suicide) (Panagioti, Gooding, \& Tarrier, 2011). In the Veteran population specifically, PTSD diagnoses and severity have likewise been shown to predict attempts (McKinney, Hirsch \& Britton, 2017). A recent meta-analysis (May \& Klonsky, 2016) highlighted PTSD as one of only a few variables associated with the likelihood that individuals who ideated would proceed to attempt. The impact of PTSD on Veterans' suicide risk seems clear; further exploration of the nature of relationship between PTSD and suicidal behavior may facilitate risk prevention efforts.

Importantly, PTSD secondary to interpersonal trauma (e.g. physical or sexual assault) has been most consistently and robustly linked to the development of suicidal behavior (Stein et al., 2010). While Veterans' trauma is often presumed to be combat- 


\section{SUICIDALITY: SEXUAL AND GENDER MINORITY CONSIDERATIONS}

related, in recent years a significant number of Veterans (22 to $45 \%$ of female Veterans and roughly $1 \%$ of males; Suris \& Lind, 2008) have endorsed exposure to MST (sexual abuse, assault, or harassment). The documented consequences of MST include myriad psychiatric diagnoses including PTSD, MDD, substance use disorders, and anxiety disorders (Kimerling, Gima, Smith, Street \& Frayne, 2007; Sexton, Raggio, McSweeney, Authier, \& Rauch, 2017), although it is worth noting that several behaviors included under the definition of MST may not satisfy Criterion A for diagnoses of PTSD.

With respect to suicidality, Bryan, Bryan and Clemans (2014) found endorsement of MST was associated with a greater risk of lifetime suicide ideation (44.8\% vs. 29.1\%), suicide plans (22.4\% vs. $11.8 \%)$, and suicide attempts (15.5\% vs. $4.9 \%)$ with a college sample of military personnel and Veterans. Among a clinical sample of Veterans with PTSD secondary to MST, Suris, Link-Malcolm and North (2011) reported depressive symptoms and PTSD hyperarousal cluster severity were unique predictors of SI. In a study of over six million Veterans Health Administration (VHA) utilizers from 2007 to 2011 tracked for suicide mortality outcomes through 2014, Kimerling, Makin-Byrd, Louzon, Ignacio and McCarthy (2016) reported MST was associated with increased risk of suicide completion among men $($ Hazard Ratio $=1.69)$ and women $($ Hazard Ratio $=$ 2.27). Thus, risk for suicidal behavior appears compounded substantially by both diagnostic (PTSD, MDD) and experiential (MST) factors, leading to a uniquely high-risk subset of the Veteran population. Yet, much of the research to date regarding MST as a suicide risk factor has focused on non-clinical Veteran samples. Clinical populations may be at even higher risk for adverse outcomes given the elevated psychiatric morbidity and greater psychological distress among those seeking mental health services. Thus, further 


\section{SUICIDALITY: SEXUAL AND GENDER MINORITY CONSIDERATIONS}

research is needed to better understand the intersection of MST and suicidality among treatment-seeking Veterans.

\section{Sexual and Gender Minorities and Suicide Risk}

Another factor capable of raising suicide risk further, both on its own and in combination with those previously noted, has received less research attention in suicide research among Veterans: sexual and gender minority (SGM) status. In population-based studies not specific to Veterans, researchers have consistently identified elevated rates of suicide attempts among SGM adolescents and adults. For instance, a meta-analysis revealed that individuals identifying as lesbian, gay or bisexual (LGB) attempt suicide at twice the rate of heterosexual peers (King et al., 2008). Rates among individuals identifying as transgender may be even higher, with as many as one third reporting a history of suicide attempts (HSA) in non-clinical samples (i.e., Clements-Nolle, Marx, \& Katz, 2006).

In recent years, researchers have increased their attention to Veterans identifying as SGM. Evidence suggests SGM status is associated with amplified risk for PTSD, lifetime exposure to sexual assault, homelessness, and legal involvement (Lehavot \& Simpson, 2013; Mattocks et al., 2013). Notably, a study by Cochran and colleagues (2013) found Veterans identifying as SGM were more likely than non-SGM peers to screen positive for mental health problems and that negative experiences during their service (e.g. stigma, bullying) related to their sexuality or gender identification were associated with greater mental health symptoms. Thus, the available evidence signals greater attention of the care needs of this population is warranted. 


\section{SUICIDALITY: SEXUAL AND GENDER MINORITY CONSIDERATIONS}

Similarly worrisome patterns have been observed in SGM Veterans with respect to both likelihood of experiencing MST and suicidal behavior. Unfortunately, those identifying as SGM are more likely to endorse MST (Mattocks et al., 2013), and many survivors indicate being targeted for assault because of their actual or perceived sexual orientation or gender status (Lehavot \& Simpson, 2014). For instance, following a survey study, Blosnich, Mays, \& Cochran (2014) indicated 22.5\% of sexual minority Veterans endorsed HSA in contrast with $7.5 \%$ of their heterosexual Veteran peers.

\section{The Current Study}

Abundant evidence supports the idea that risk for suicide is increased among Veterans with PTSD, to a greater degree among those with MST, and among SGM individuals. The aim of the present study is to fill gaps in the literature concerning lifetime suicide attempt risk in sexual and gender minorities, specifically, and among Veterans seeking clinical interventions associated with MST more broadly. We hypothesized that SGM Veterans would endorse lifetime HSA at significantly higher rates than their non-SGM peers despite both groups endorsing exposure to MST. We further sought to characterize the overall HSA risk for Veterans presenting for MSTrelated care as previous research has been limited to non-clinical populations. In so doing, our intentions were to ascertain whether these contextual factors present heightened risk factors warranting further empirical and clinical attention within the VHA's greater efforts to combat suicide and to facilitate the reduction of healthcare disparities within empirical assessments of Veterans' mental health and care needs.

\section{Method}

\section{Participants and Procedures}




\section{SUICIDALITY: SEXUAL AND GENDER MINORITY CONSIDERATIONS}

Veterans seeking MST-related treatment at a Midwestern Veterans Healthcare Administration (VHA) hospital presented for assessment and liaison to appropriate care. Between 2010-2015, 277 Veterans attended an initial interview incorporating psychosocial histories and clinical assessment of mental health symptoms. Participants were primarily female (70.8\%) and Caucasian (71.3\%) with an average age of 44.4 years $(S D=12)$. A variety of service eras were represented, though only a minority was exposed to combat (18.5\%). Of those identifying as SGM, eight Veterans self-described as lesbian, six as gay, six as bisexual, and one as transgender. In addition, nine described themselves as SGM but did not further characterize their minority sexual and gender status. Demographic characteristics are further detailed in Table 1.

At intake, Veterans completed a semi-structured interview (Mini-International Neuropsychiatric Interview [MINI] Sheehan et al., 1998) to assess for the presence of a variety of clinical sequelae including PTSD symptoms, mood disorders, anxiety disorders, and substance use problems as well as lifetime HSA in order to facilitate triage to appropriate interventions. Demographic information, including sexual orientation and gender identification, were assessed and documented in the evaluation report.

An electronic chart review template was used to code information to confirm that MST was the focal event for which care was being sought and to capture demographic and military characteristics and diagnostic findings from the clinical interview. Providers with specialty training in the assessment and treatment of MST and/or PTSD were trained on the data extraction procedure. Upon completion of the chart reviews, data were coded and fidelity checks were performed. The hospital's Institutional Review Board and the 


\section{SUICIDALITY: SEXUAL AND GENDER MINORITY CONSIDERATIONS}

Health Services Research and Development Board approved the processes and procedures involved in this research.

\section{Materials and Measures}

\section{Demographic characteristics and sexual orientation and gender self-}

identification. Demographic characteristics including sexual orientation and transgender identification were initially assessed on self-report intake paperwork. Responses were reviewed during the clinical interview and incorporated into clinical care as warranted.

Psychiatric morbidity and history of suicide attempts. The Mini-International Neuropsychiatric Interview-IV (MINI; Sheehan et al., 1998) is a semi-structured interview of Diagnostic and Statistical Manual-IV (DSM-IV; American Psychiatric Association) criteria including current symptoms of PTSD, mood, anxiety, and alcohol and other substance use disorders. In addition, past-month and lifetime HSA were assessed separately and distinctly from engagement in behaviors considered to be instances of non-suicidal self-injury.

\section{Data Analysis}

Demographic and mental health characteristics were analyzed using means, percentages, and parametric and non-parametric between-group contrasts as warranted. Chi-square analysis was used to compare SGM and non-SGM groups on lifetime HSA. The $a$ priori alpha was established at $p<.05$ and Cramer's $\Phi$ was used to characterize the magnitude of the effect. All analyses were conducted with SPSS 22.0.

\section{Results}

As would be anticipated in a treatment-seeking population, psychiatric disorders were common with PTSD (80.9\%) and mood (75.9\%) disorders the most frequently 


\section{SUICIDALITY: SEXUAL AND GENDER MINORITY CONSIDERATIONS}

diagnosed. Regarding sexual and gender minority identification, 10.1\% identified as SGM $(n=28)$. Chi-square and $t$-test analyses found SGM and non-SGM groups did not differ significantly on demographic or military characteristics or prevalence of psychiatric diagnoses (see Table 1).

Overall, $30.7 \%$ of Veterans endorsed lifetime HSA. Rates of HSA were nearly twice as high among the SGM group (53.6\%) than the non-SGM group (28.1\%). Chisquare analyses indicated the difference was statistically significant $\left(\chi^{2}=7.67, d f=1, p=\right.$ .006). Estimates suggested the magnitude of the effect was small-to-moderate in size (Cramer's $\Phi=.166)$.

\section{Discussion}

This study examined the suicidal behavior and psychiatric presentations of sexual and gender minority treatment-seeking Veterans with a history of MST. This population of Veterans represents a group facing multiple minority identities - survivors of MST and people who identify as a sexual or gender minority. In spite of finding no differences in the rates of psychiatric disorders, SGM in this sample of Veterans were far more likely to endorse lifetime rates of HSA. Indeed, rates of HSA were nearly twice as high among SGM (53.6\%) treatment-seeking Veterans than non-SGM MST treatment-seeking Veterans (28.1\%).

Our results are consistent with other research finding high rates of HSA in MST survivors (Bryan et al., 2015), among SGM civilians (King et al., 2008) and SGM Veterans (Blosnich et al., 2014) compared to non-SGM peers. Yet, this is one of the first studies to examine HSA in MST treatment-seeking SGM veterans. Our findings that the frequency of HSA endorsed by our sample was demonstrably higher than those 


\section{SUICIDALITY: SEXUAL AND GENDER MINORITY CONSIDERATIONS}

documented in prior research is troubling and perhaps due, in part, to synergistic interactions of trauma history, mental health presentations, and minority statuses. This suggests that estimates based on non-clinical samples do not appear to generalize to this group. One explanation may be that Veterans seeking treatment may elect to do so at the peak of distress and when symptoms are significant.

It is interesting that we did not find differences in rates of psychiatric disorders between SGM and non-SGM groups exposed to MST. This is inconsistent with research in SGM civilians (King et al., 2008) and SGM Veterans (Lehavot \& Simpson, 2013). It may be that those who identify as SGM may be at greater risk for developing negative psychiatric sequlae, but that among those seeking care their clinical presentations are similar to non-SGM survivors of MST. It may also be the case that the prevalence of clinical disorders are similar but that groups may differ in terms of severity, although this possibility was not assessed in our study. It is further important to stress the high levels of PTSD, MDD, and substance use morbidities present in this sample given their importance for suicide risk assessment and management.

Given the amplified risk for suicide attempts among those exposed to MST and those identifying as SGM, attention is warranted to better understand these contextual characteristics. One theory that could provide some explanation for the elevated HSA risk within this population is the Interpersonal Theory of Suicide (Joiner, 2005). This theory proposes three constructs that predict suicidal behaviors: thwarted belongingness, perceived burdensomeness, and acquired capability. Among females exposed to MST, Montheith, Bahraini, and Menefee (2017) found these three factors were correlated with past week SI and that perceived burdensomeness and fearlessness about death retained 


\section{SUICIDALITY: SEXUAL AND GENDER MINORITY CONSIDERATIONS}

significance in their model even after adjusting for HSA and symptoms of depression. Similar, SGM Veterans are likely to be a population at high risk for these features. For instance, military culture and experiences may increase comfort with and access to weapons, habituation to violence or death, and difficulties reintegrating into society. Values traditionally associated with military culture including strength and resiliency, and potential stigmas around mental health symptoms or seeking care could contribute to increased perceived burdensomeness in Veterans who are struggling. Similarly, traumatized Veterans may exhibit numbing symptoms that reduce fear of death (Skopp, Luxton, Bush \& Rudd, 2011). These factors may lead to an increased capability. In addition, those identifying as SGM may be at greater risk for lack of acceptance by family, fellow service members, or religious groups before, during, and after service (e.g. Lehavot \& Simpson, 2013). Indeed, sexual minority women Veterans report deterioration of social support and internalized self-stigma both during and following service (Lehavot \& Simpson, 2013), which is suggestive of high levels of thwarted belongingness. The confluence of military service, trauma exposure, and SGM status may accompany especially high risk.

The finding that slightly over half of the SGM Veterans endorsed lifetime HSA can also be seen as consistent with the interpersonal theory of suicide. In particular, this population may be at disproportionately high risk for feelings of thwarted belongingness and perceived burdensomeness, as well as heightened levels of acquired capability given their training, military experiences, and sociohistorical stressors associated with SGM status within military and civilian contexts. Further research is needed to evaluate these constructs with this population. 


\section{SUICIDALITY: SEXUAL AND GENDER MINORITY CONSIDERATIONS}

Given these high rates of HSA, preventative and risk reduction strategies during service are highly recommended. Following directly from interpersonal theories of suicide, practices within the armed services to facilitate unit cohesion while respecting individual differences related to gender and sexual orientation diversity could reduce the risk of suicide for SGM Veterans. Indeed, being "out" may increase social cohesion in units (Chaplo \& Bennett, 2016). Perpetration of sexual violence is more likely when it is perceived as peer-normative (Thompson, Swartout, \& Koss, 2013); reducing this norm would be an additional intervention target that would also likely increase unit cohesion and, potentially, decrease risk of MST. Interventions to facilitate unit cohesion and reduce positive peer norms around violence are consistent with military priorities on suicide prevention.

Results highlight the high importance of thorough suicide risk assessment among Veterans with histories of MST and, perhaps especially, among individuals identifying as SGM seeking mental health care. VHA clinical practice guidelines should be followed to evaluate for the determination of suicide risk level (i.e. low, moderate, high) and matching safety planning and care to the Veteran's current needs (i.e. hospitalization, crisis management, liaison to cognitive therapy or problem-solving therapies to treat specifically for suicide risk). Additionally, as part of the suicide risk assessment process with individuals identifying as SGM, clinicians should be sure to consider the individual's social support, outness, and physical and emotional safety. Particularly in cases where Veterans may not have an established LGBT support community, provider awareness of regional connections may facilitate connection to social supports such as 


\section{SUICIDALITY: SEXUAL AND GENDER MINORITY CONSIDERATIONS}

sexual and gender minority events (i.e. Pride Month activities), LGBT Veteran peers if available, or LGBT support groups within the VA and community.

Among Veterans who are at lower to moderate risk, treatment of primary disorders such as PTSD may serve as a buffer against recurrence of suicidal ideation and behaviors. Trauma-focused interventions such as Prolonged Exposure Therapy and Cognitive Processing Therapy have been shown to reduce symptoms of PTSD and comorbid depression (Eftekhari et al. 2013; Suris, Link-Malcolm, Chard, Ahn, \& North, 2013) and, importantly, to decrease suicidal ideation in studies of survivors of sexual traumas (Cox et al., 2016) although further research is needed to evaluate the clinical effectiveness of these interventions with Veterans identifying as SGM.

Creating supportive therapeutic environments may also be paramount to proper assessment and care as research suggests that only 33\% of SGM minorities report their sexual status to health care providers, and 25\% avoid seeking VHA services (including mental health service) for fear of discrimination (Simpson, Balsam, Cochran, Lehavot \& Gold, 2013). In these numerous and likely synergistic ways, SGM Veterans who have experienced MST are at increased risk for HSA. We recommend that clinicians start with simple steps such as asking and using the patient's preferred name and pronoun; we consider sexual orientation/gender identity to be as critical to care as other demographic indicators (Chaplo \& Bennett, 2016). Case conceptualization should include consideration of the intersection between gender identity and sexual orientation with other demographic and clinical characteristics and SGM-affirmative approaches to patient care are strongly recommended. For instance, Proujanski \& Pachankis, (2014) have provided evidence-based suggestions to detail explicit methods of utilizing sexual 


\section{SUICIDALITY: SEXUAL AND GENDER MINORITY CONSIDERATIONS}

minority -affirming principles within standard care delivery that can be integrated within existing evidence-based care. Specifically, attending to negative mental health sequalae of minority stress, augmenting emotional awareness and regulation skills, countering avoidance behaviors that may impede Veterans' clinical goals, facilitating restructuring of negative cognitions specific to minority stress (i.e. thoughts associated with internalized homophobia or gender stereotypes), improving assertive communication to facilitate client empowerment, and validating and strengthening individual resilience.

Augmenting practitioner proficiencies and consideration of systemic and structural change is also warranted. The provision of internal initiatives to increase awareness and cultural competency may include training for clinicians as well as review of clinic and hospital forms to ensure use of inclusive language, for example. Exploration of barriers to VHA service utilization in high-risk populations, (e.g. SGM, MST exposure) must be prioritized moving forward. In addition, we encourage facilities to have LGBT support or peer-to-peer groups, display SGM-tailored outreach materials throughout facilities to promote inclusivity among representations of Veteran care seekers and increase awareness of SGM-affirmative practices and resources, and encourage providers to be visible at community or hospital events such as holding Pride events during LGBT Pride Month or having VHA information tables at events held within the community. In addition, consideration of factors important to delivering healthcare to SGM identified Veterans within continued education is recommended such as explicit consideration at case conference presentations or colloquium.

Our results should be interpreted in light of the study's limitations. For instance, the cross-sectional methodology and assessment of lifetime HSA cannot speak to the time 


\section{SUICIDALITY: SEXUAL AND GENDER MINORITY CONSIDERATIONS}

period in which suicide attempts occurred. Further, the sample size of the current study precluded the examination of specific subpopulations within the SGM Veteran community, which could be further illustrative for more precisely targeting risk reduction efforts. Research with larger sample sizes would further permit assessment of potential moderating and interactive factors that may improve our ability to recognize and respond to the needs of survivors. Finally, many of the variables examined in this study are sensitive in nature (SGM status, MST, psychiatric symptoms, suicidal behaviors). It is impossible to know if issues around disclosure, or lack thereof, influenced reporting of these characteristics at initial clinical visits.

Despite these limitations, this study has several strengths that are worth noting. In particular, this study reduces some of the health disparities that are known to exist in the empirical literature by focusing on several understudied populations, including SGM, female Veterans, and service members that have experienced MST. The focus on HSA is particularly important given that previous attempts are among the most reliable predictors of completed suicide (Joiner, 2005). Similarly, while we acknowledge that the results may not generalize to people who are not seeking treatment, much of the previous research has focused on non-clinical populations. To our knowledge, this is the first study to examine HSA among a clinical sample of Veterans exposed to MST of any sexual or gender status and also the first to evaluate whether identification as SGM is associated with greater risk of attempts among treatment-seekers. Our investigation of treatmentseeking Veterans adds to the literature and offers important information and insights for clinicians who will be tasked with providing care. Finally, the assessment of concurrent mental health diagnoses in this study demonstrates that the differences in HSA histories 


\section{SUICIDALITY: SEXUAL AND GENDER MINORITY CONSIDERATIONS}

between those identifying as SGM or non-SGM cannot be solely attributed to dissimilarities in diagnostic presentations as the psychiatric morbidity rates among groups were similar.

While the current study adds to the extant literature, broader aspects of suicidality, including suicidal ideation, suicide completion, repeated attempts, and timeline characteristics of suicidal behavior should be explored. In addition, previous literature has demonstrated that certain event characteristics of MST experienced by SGM may differ from non-SGM (Lehavot \& Simpson, 2014). Studies are also needed to examine if dual exposure to MST and other types of traumas (i.e. childhood trauma, combat exposure, non-military sexual or physical trauma in adulthood) or contextual elements of the MST events have main or interactive relationships with risk behaviors.

\section{Conclusions}

SGM Veterans likely face multiple challenges related to minority stress as part of their service. Our study found that the majority of SGM Veterans seeking MST care experienced HSA, at double the rate of suicide attempts compared to their non-SGM peers. Similar, among all Veterans seeking MST-related care, our clinical sample endorsed HSA at twice the rate as non-treatment seekers exposed to MST. This underscores a need for greater preventive efforts and clinical attention to the needs of SGM Veterans and MST survivors to reduce these serious health disparities. 


\section{SUICIDALITY: SEXUAL AND GENDER MINORITY CONSIDERATIONS}

\section{References}

Blosnich, J. R., Mays, V. M., \& Cochran, S. D. (2014). Suicidality among veterans: implications of sexual minority status. American Journal of Public Health, 104(S4), S535-S537. doi: 10.2105/AJPH.2014.302100

Bryan, C.J., Bryan, A. O., \& Clemans, T. A. (2014). Gender differences in the association of military sexual trauma with suicide risk [American Psychological Association Press release]. Retrieved from https://www.apa.org/news/press/releases/2014/08/military-sexual-trauma.pdf

Chaplo, S. D., \& Bennett, D. C. (2016). Veterans. In A. Goldberg (ed.), Sage Encyclopedia of LGBTQ Studies. Los Angeles, CA: Sage.

Clements-Nolle K, Marx R, Katz M. (2006). Attempted suicide among transgender persons: The influence of gender-based discrimination and victimization. Journal of Homosexuality, 51, 53-69. doi: /10.1300/J082v51n03_04

Cochran, B. N., Balsam, K., Flentje, A., Malte, C. A., \& Simpson, T. (2013). Mental health characteristics of sexual minority veterans. Journal of Homosexuality, 60(2-3), 419-435. doi: 10.1080/00918369.2013.744932

Cox, K. S., Mouilso, E. R., Venners, M. R., Defever, M. E., Duvivier, L., Rauch, S. A., ... \& Tuerk, P. W. (2016). Reducing suicidal ideation through evidence-based treatment for posttraumatic stress disorder. Journal of Psychiatric Research, 80, 59-63. doi: 10.1016/j.jpsychires.2016.05.011

Eftekhari, A., Ruzek, J. I., Crowley, J. J., Rosen, C. S., Greenbaum, M. A., \& Karlin, B. E. (2013). Effectiveness of national implementation of prolonged exposure 


\section{SUICIDALITY: SEXUAL AND GENDER MINORITY CONSIDERATIONS}

therapy in Veterans Affairs care. JAMA Psychiatry, 70(9), 949-955. doi: 10.1001/jamapsychiatry.2013.36

Huguet, N., Kaplan, M. S., \& McFarland, B. H. (2014). The effects of misclassification biases on veteran suicide rate estimates. American Journal of Public Health, 104(1), 151-155. doi: 10.2105/AJPH.2013.301450

Joiner, T (2005). Why People Die by Suicide. Harvard University Press, Cambridge, MA.

Kimerling, R., Gima, K., Smith, M. W., Street, A., \& Frayne, S. (2007). The Veterans Health Administration and military sexual trauma. American Journal of Public Health, 97(12), 2160-2166. doi: 10.2105/AJPH.2006.092999

Kimerling, R., Makin-Byrd, K., Louzon, S., Ignacio, R. V., \& McCarthy, J. F. (2016). Military sexual trauma and suicide mortality. American Journal of Preventive Medicine, 50(6), 684-691. doi: 10.1016/j.amepre.2015.10.019

King, M., Semlyen, J., Tai, S. S., Killaspy, H., Osborn, D., Popelyuk, D., \& Nazareth, I. (2008). A systematic review of mental disorder, suicide, and deliberate self harm in lesbian, gay and bisexual people. BMC Psychiatry, 8(1), 70. doi: 10.1186/1471$244 X-8-70$

Lehavot, K., \& Simpson, T. L. (2013). Incorporating lesbian and bisexual women into women veterans' health priorities. Journal of General Internal Medicine, 28(2), 609-614. doi: 10.1007/s11606-012-2291-2

Lehavot, K., \& Simpson, T. L. (2014). Trauma, posttraumatic stress disorder, and depression among sexual minority and heterosexual women veterans. Journal of Counseling Psychology, 61(3), 392. doi: 10.1037/cou0000019 


\section{SUICIDALITY: SEXUAL AND GENDER MINORITY CONSIDERATIONS}

Mattocks, K. M., Sadler, A., Yano, E. M., Krebs, E. E., Zephyrin, L., Brandt, C., ... \& Allison, J. (2013). Sexual victimization, health status, and VA healthcare utilization among lesbian and bisexual OEF/OIF veterans. Journal of General Internal Medicine, 28(2), 604-608. doi: 10.1007/s11606-013-2357-9

May, A. M., \& Klonsky, E. D. (2016). What distinguishes suicide attempters from suicide ideators? A meta-analysis of potential factors. Clinical Psychology: Science and Practice. doi: 10.1111/cpsp.12136

McKinney, J. M., Hirsch, J. K., \& Britton, P. C. (2017). PTSD symptoms and suicide risk in veterans: Serial indirect effects via depression and anger. Journal of Affective Disorders, 214, 100-107. doi: 10.1016/j.jad.2017.03.008

Monteith, L. L., Bahraini, N. H., \& Menefee, D. S. (2017). Perceived burdensomeness, thwarted belongingness, and fearlessness about death: associations with suicidal ideation among female veterans exposed to military sexual trauma. Journal of Clinical Psychology. doi: 10.1002/jclp.22462

Panagioti, M., Gooding, P., \& Tarrier, N. (2009). Post-traumatic stress disorder and suicidal behavior: A narrative review. Clinical psychology review, 29(6), 471-482. doi: 10.1016/j.cpr.2009.05.001

Proujansky, R. A., \& Pachankis, J.E. (2014). Toward formulating evidence-based principles of LGB-affirmative psychotherapy. Pragmatic Case Studies in Psychotherapy, 10(2), 117-131.

Sexton, M. B., Raggio, G. A., McSweeney, L. B., Authier, C. C., \& Rauch, S. A. (2017). Contrasting gender and combat versus military sexual traumas: Psychiatric 


\section{SUICIDALITY: SEXUAL AND GENDER MINORITY CONSIDERATIONS}

symptom severity and morbidities in treatment-seeking veterans. Journal of Women's Health. Advance online publication. doi:.1089/jwh.2016.6080

Sheehan, D., Lecrubier, Y., Sheehan, K. H., Sheehan, K., Amorim, P., Janavs, J., et al. (1998). Diagnostic Psychiatric Interview for DSM-IV and ICD-10. Journal of Clinical Psychiatry, 59, 22-33.

Simpson, T. L., Balsam, K. F., Cochran, B. N., Lehavot, K., \& Gold, S. D. (2013). Veterans administration health care utilization among sexual minority veterans. Psychological Services, 10(2), 223. doi: 10.1037/a0031281

Skopp, N. A., Luxton, D. D., Bush, N., \& Rudd, D. (2011). Managing the Service Member with Suicidal Ideation. In B.A. Moore, \& W.E. Penk, (Eds.), Handbook for the treatment of PTSD in Military Personnel (288-304). New York: The Guilford Press.

Surís, A., \& Lind, L. (2008). Military sexual trauma: A review of prevalence and associated health consequences in veterans. Trauma, Violence, \& Abuse, 9(4), 250269. doi: $10.1177 \% 2 F 1524838008324419$

Surís, A., Link- Malcolm, J., Chard, K., Ahn, C., \& North, C. (2013). A randomized clinical trial of cognitive processing therapy for veterans with PTSD related to military sexual trauma. Journal of Traumatic Stress, 26(1), 28-37.

Surís, A., Link-Malcolm, J., \& North, C. S. (2011). Predictors of suicidal ideation in veterans with PTSD related to military sexual trauma. Journal of Traumatic Stress, 24(5), 605-608. doi: 10.1002/jts.20674 


\section{SUICIDALITY: SEXUAL AND GENDER MINORITY CONSIDERATIONS}

Thompson, M. P., Swartout, K. M., \& Koss, M. P. (2013). Trajectories and predictors of sexually aggressive behaviors during emerging adulthood. Psychology of Violence, 3(3), 247-259. doi: 10.1037/a0030624

Weiner, J., Richmond, T. S., Conigliaro, J., \& Wiebe, D. J. (2011). Military veteran mortality following a survived suicide attempt. BMC Public Health, 11(1), 374. doi: $10.1186 / 1471-2458-11-374$ 
Table 1.

Participant Demographic, Military, and Psychiatric Characteristics $(N=277)$

\section{SM (M or \%) N-SM (M or \%)}

Characteristic $n=28 \quad n=249 \quad t$ or $\chi^{2} \quad p$

Demographic Characteristics

Female

$57.1 \%$

$72.3 \%$

$2.791 \quad .095$

Caucasian

$67.9 \%$

$71.7 \%$

$0.178 \quad .673$

Age

$45.8(12.0)$

$44.2(12.5)$

$-.644 \quad .520$

Service Era

$1.524 \quad .677$

Vietnam War

$14.3 \%$

$16.1 \%$

Post-Vietnam

$32.1 \%$

$23.3 \%$

Persian Gulf

$39.3 \%$

$39.0 \%$

OIF/OEF/OND

$14.3 \%$

$21.7 \%$

Combat Experience

$11.5 \%$

$19.3 \%$

0.936

.333

Psychiatric Morbidity

PTSD

Mood Disorder

Anxiety Disorder

Substance Use Disorder
$75 \%$

$81.6 \%$

0.603

.437

$79.2 \%$

$75.6 \%$

$0.152 \quad .696$

$37.5 \%$

$31.8 \%$

0.321

.571

$29.2 \%$

$0.260 \quad .610$

Notes: $\mathrm{SM}=$ Sexual Minority Identification; N-SM = Non-Sexual Minority

Identification; OIF/OEF/OND = Operation Iraqi Freedom/Operation Enduring

Freedom/Operation New Dawn Era; PTSD = Posttraumatic Stress Disorder. 